\title{
VARIACIÓN MORFOMÉTRICA EN RESTOS ÓSEOS DE GUANACO DE SITIOS ARQUEOLÓGICOS DE PATAGONIA AUSTRAL CONTINENTAL Y DE LA ISLA GRANDE DE TIERRA DEL FUEGO
}

\author{
GABRIELA L. L'HEUREUX*
}

\begin{abstract}
RESUMEN
El objetivo de este trabajo es evaluar la variación morfológica de restos óseos de Lama guanicoe en ambos lados del estrecho de Magallanes, a partir del análisis osteométrico de elementos del esqueleto apendicular. Los huesos analizados en este trabajo son: falanges -primeras y segundas-, húmeros distales y metacarpos distales. En el análisis se utilizaron muestras provenientes de 6 sitios arqueológicos y 1 paleontológico del sector más austral de Patagonia continental y de 4 sitios arqueológicos de la isla grande de Tierra del Fuego. Se compararon dos períodos temporales: a) Pleistoceno final (ca. 12.000 - 10.000 años AP), y b) Holoceno tardío final (ca. 2.000 AP hasta el presente). Se aplicaron técnicas estadísticas uni y multivariadas para el análisis de los datos. Los resultados obtenidos mostraron tamaños similares en las $2^{\text {as }}$ falanges del Pleistoceno final de Patagonia continental austral y de Tierra del Fuego, y tamaños significativamente diferentes entre ambas regiones en todos los elementos analizados del Holoceno tardío final. Esta diferencia se traduce en la presencia de tamaños más grandes y formas más robustas en los elementos de guanaco procedentes de Tierra del Fuego. Se considera que Patagonia austral continental e insular representan casos particulares desde un punto de vista histórico y biogeográfico, en el que convergen diferentes causas y procesos. Para Tierra del Fuego, hay un factor influyente que puede explicar el patrón observado: el efecto de aislamiento producido ca. 8.000 años AP y el consecuente proceso de evolución divergente de las poblaciones animales que habitaban el área ancestral.
\end{abstract}

PALABRAS CLAVES: morfometría de guanaco, Patagonia austral continental e insular, Pleistoceno final, Holoceno tardío.

\section{MORPHOMETRIC VARIATION OF GUANACO BONE REMAINS FROM ARCHAEOLOGICAL SITES OF SOUTHERN PATAGONIA AND TIERRA DEL FUEGO}

\begin{abstract}
The aim of this paper is to evaluate the morphological variation of Lama guanicoe appendicular bone remains recovered from either side of the Magellan strait using a traditional morphometric approach. The analyzed bones are: 1st and 2nd phalanges, distal humeri, and distal metacarpals. The samples are from 6 archaeological

* CONICET. Departamento de Investigaciones Prehistóricas y Arqueológicas, IMHICIHU. Saavedra 15, piso 5a, (1083 ACA) Capital Federal, Argentina. E-mail: dipa.imhicihu@ conicet.gov.ar
\end{abstract}


and 1 paleontological sites from southern Patagonia, and 4 sites from the Isla Grande of Tierra del Fuego. Two temporal periods were compared: a) Late Pleistocene (ca. $12.000-10.000{ }^{14} \mathrm{C}$ years $\mathrm{BP}$ ), and b) Late Holocene (ca. $2.000{ }^{14} \mathrm{C}$ years BP up to the present). Univariate and multivariate statistical techniques were applied. The results obtained show no significant difference in size between Late Pleistocene samples from southern Patagonia and Tierra del Fuego at the level of the 2nd phalanges. In contrast, the comparison of Late Holocene samples from both regions show significant differences in size in all the appendicular elements analyzed. In the latter case, the larger and more robust elements are those from Tierra del Fuego. It is concluded that such differences can be considered as an evidence of the reproductive isolation and vicariance of guanaco populations from both sides of the Magellan Strait occurred after $8.000{ }^{14} \mathrm{C}$ years BP.

KEY WORDS: guanaco morphometrics, southern Patagonia and Tierra del Fuego, Late Pleistocene, Late Holocene.

\section{INTRODUCCIÓN}

Uno de los problemas que surgen al comparar conjuntos óseos de guanaco de diferentes latitudes -y que presentan comportamientos morfológicos disímiles-, es la identificación de las fuentes de variación de tales estructuras morfológicas. Si dejamos de lado la variación temporal (utilizando muestras promediadas de unidades estratigráficas que presentan fechados radiocarbónicos comparables), las fuentes de variación geográfica pueden relacionarse con: a) factores bióticos: los tamaños de las presas; competencia; preación; abundancia y tipo de recursos; deriva génica y efecto fundador, etc. (Forsman 1991; McNab 1971; Rosenzweig 1966; Ronquist y Nylin 1990). b) abióticos: efectos del clima regulados por la latitud y la altitud (Regla de Bergmann: Ashton et al. 2000; Brown y Lee 1969; Davis 1981; James 1970); duración de la época de crecimiento (Roff 1980); formación de una nueva barrera geográfica (vicarianza) - aislamiento (Ronquist 1997; Rosen 1978; Wiley 1988). c) la combinación de ambos.

El tamaño corporal de cualquier organismo tiene importantes implicancias para su fitness, para el uso que hace de los recursos, la relación predador-presa que establece y la historia biológica del área en que habita (Roff 1992; Atkinson 1994). Se conoce que el tamaño del cuerpo muestra variaciones geográficas intraespecíficas importantes y no asociadas al dimorfismo sexual (Ashton et al. 2000). Una de las reglas biogeográficas más conocidas al respecto, es la regla de Bergmann (Bergmann 1847). Ésta asume que el incremento en el tamaño del cuerpo de poblaciones homeotermas se correlaciona positivamente con el incremento de la latitud. En el presente caso, consideramos que tener en cuenta dicha regla en la interpretación de los datos no es adecuado, en tanto no se analiza una distribución latitudinal amplia de las muestras, y que, de ser así, se necesitaría una revisión profunda del tema debido a los cuestionamientos surgidos en la verificación de la regla en mamíferos, principalmente aquéllos de gran tamaño (Hayward 1956; Mousseau 1997; Ray 1960; Scholander 1955, contra Ashton et al. 2000).

Una de las explicaciones que se ajustaría mejor al caso de estudio es la existencia de un proceso de vicarianza y posterior evolución divergente. La vicarianza sostiene que la existencia de taxas con patrones bifurcados resulta de la imposición de cambios en la distribución continua del taxa originario. Estos cambios se relacionan con la formación de barreras naturales (p.e. formación de una cadena montañosa; insularización, etc.), que dividen el área ocupada ancestralmente. Este proceso tiene la particularidad de involucrar a numerosos taxa, los cuales deben adaptarse a situaciones y presiones diferentes a las sostenidas previamente. En un caso de vicarianza la disyunción se forma en el mismo momento que la barrera aparece, y a partir de este evento, cada población puede evolucionar independientemente (Crici y Morrone 1990; Platnick y Nelson 1978; Wiley 1988). En otras palabras, la existencia de Lama guanicoe en áreas biogeográficas independientes, se explica por vicarianza considerando que sus ancestros ocupaban un espacio único que comprendía las actuales áreas disyuntas, las cuales representan en el presente los vestigios de la distribución ancestral. Existe evidencia arqueológica que confirma la presencia de Lama guanicoe desde por lo menos 12.000 años AP en la isla grande de Tierra 
del Fuego y en el extremo austral del continente (Borrero 2003; Borrero et al. 1997; Massone 1987; Massone et al. 1999; Prieto 1991).

\section{ESCENARIO PASADO Y ACTUAL EN PATAGONIA AUSTRAL}

Para enmarcar la presente investigación, es importante hacer una revisión de los procesos paleoclimáticos generales y las modificaciones geográficas producidas durante los últimos 12.000 años en el extremo de Patagonia austral. El área de estudio cuenta con un panorama paleoambiental caracterizado principalmente por condiciones climáticas fluctuantes y por una flexibilidad en las transformaciones de los rasgos geográficos y su distribución (retrocesos y reavances de los glaciares de montaña, recuperación de los bosques, vulcanismo, aumento del nivel del mar, etc.), lo que acarreó importantes consecuencias en la distribución, expansión y disponibilidad de los diferentes ambientes y de los recursos vegetales y animales (Borrero 2001; Rabassa et al. 1992a).

El Pleistoceno final en Tierra del Fuego se distinguió por condiciones climáticas rápidamente cambiantes que culminaron en el retroceso del Glacial Beagle y el desarrollo de un frente glacial parcialmente flotante en un lago proglacial o en el mar transgresivo. El hielo glaciario aún estaba presente en el Canal del Beagle y en partes del estrecho de Magallanes hacia el 10.000 AP en forma de glaciares activos en retroceso o muertos en procesos de desintegración. El límite Pleistoceno - Holoceno definido en los 10.000 años radiocarbónicos AP coincide con la recesión final del hielo y con el rápido desarrollo de los ecosistemas forestales en Tierra del Fuego (Rabassa et al. 1992a).

Alrededor de los 9.000 años AP el mejoramiento climático registrado, impuso condiciones algo más cálidas que las actuales hasta ca. 6.500 AP. Estos cambios climáticos se correlacionaron con numerosas transformaciones geológicas: se forma el estrecho de Magallanes mediante el proceso de aumento de la temperatura -derretimiento de los hielos-, aumento del nivel del mar (Borrero 2001; McCulloch et al. 1997). El ambiente marino se estableció en forma permanente a lo largo del canal y probablemente en toda la periferia de la isla grande de Tierra del Fuego hacia el 7.600 AP con una elevación relativa máxima del nivel del mar alrededor del 6.000 AP
(Rabassa et al. 1992a). Con el retiro de las aguas de la transgresión Flandrian (ca. 5.000 AP), se inició un cambio en la geografía de las costas: en Tierra del Fuego empezó a formarse el istmo que separó las bahías de San Sebastián e Inútil, y en el extremo sur de Patagonia continental comenzó a formarse la Punta Dungeness (Rabassa et al. 1992b).

Aproximadamente después del 5.000 AP, ya quedaron fijados los límites contemporáneos de los ecotonos bosque-estepa (Markgraf 1989), aunque estudios centralizados en columnas polínicas recientes identificaron alguna variación. Hacia los 2.000 años $\mathrm{AP}$, numerosa evidencia muestra que en Patagonia continental austral, la secuencia climática siguió con una tendencia general hacia la aridez (proceso iniciado a partir del 6.000 AP), aunque con marcadas fluctuaciones climáticas (Markgraf 1988; Stine y Stine 1990; Rabassa et al. 1992b).

En la actualidad, en el extremo austral de la $\mathrm{Pa}-$ tagonia continental y en Tierra del Fuego, predominan condiciones climáticas semejantes, aunque hacia el sur la media en la temperatura va disminuyendo y por la latitud $50-55^{\circ} \mathrm{S}$ los vientos se vuelven más severos y los veranos más cortos (Rabassa y Clapperton 1990). En el sector más austral de Patagonia continental, como remanente de la última glaciación, se registra un gran campo de hielo al pie de la Cordillera de los Andes: el Hielo Patagónico Sur, de $13.000 \mathrm{Km}^{2}$ de extensión. En Tierra del Fuego, también subsiste un gran campo de hielo centrado en la cordillera Darwin, el cual presenta abundantes glaciares que descargan en el estrecho de Magallanes y en el canal del Beagle (Rabassa y Clapperton 1990). Estas capas de hielo, son consideradas importantes barreras y tuvieron -y tienen- un importante rol en la circulación de las poblaciones humanas y de animales al este de estas cadenas montañosas.

A partir de lo brevemente desarrollado arriba, podemos concluir que contamos con muestras procedentes de una región que sufrió importantes transformaciones geológicas y ambientales durante los últimos 12.000 años, la cual debe ser estudiada en consecuencia teniendo en cuenta la información geológica y paleoambiental disponible en escala regional y/o local. En principio, contamos con muestras procedentes de dos regiones en momentos en que ambas formaban parte de un mismo territorio (ca. 12.000 - 10.000 años AP). Como se mencionó, el mismo no era continuo y no estaba completamente 
disponible debido a la presencia de grandes cuerpos de hielo y glaciares que subsistieron hasta comienzos del Holoceno. Por otro lado, tenemos muestras de esas mismas regiones en un momento en que dichas áreas ya no formaban parte del mismo territorio (después del 2.000 AP), sino que representan formaciones geológicas independientes que llevan separadas más de 6.000 años.

\section{MUESTRA ANALIZADA}

Para este trabajo se midieron los elementos óseos de siete sitios de Patagonia austral continental (áreas de Última Esperanza -Chile-, Sierra Baguales, Pali Aike y la cuenca del río Gallegos -Argentina-), y cuatro sitios de la isla grande de Tierra del Fuego (áreas de la cuenca del río San Martín, Bahía San Sebastián -territorios argentino y chileno-) (Fig. 1).

En el conjunto arqueológico considerado para Patagonia continental, se analizaron los sitios: Cueva Lago Sofía 1 (Prieto 1991); Chorrillo Malo 2 (Franco 2002; Franco y Borrero 2003; Franco et al. 1999); Co. Verlika 1 (Franco 2002; Franco et al. 1999); Co. Verlika 3 (Franco et al. 1999; L'Heureux 1999a); Las Buitreras (Caviglia y Figuerero Torres 1976; Sanguinetti de Bórmida 1976, 1980, 1999); Cabo Vírgenes 8 (Borrero y Franco 1999; L'Heureux 1999b). El único sitio paleontológico incorporado fue Cueva Lago Sofía 4 (Prieto 1991; Borrero et al. 1997).

TABLA 1. Sitios analizados. Unidades estratigráficas consideradas y sus cronologías.

\begin{tabular}{|c|c|c|c|c|}
\hline \multirow{2}{*}{$\begin{array}{c}\text { SITIOS } \\
\text { ARQUEOLÓGICOS }\end{array}$} & \multirow{2}{*}{$\begin{array}{c}\text { UNIDAD } \\
\text { ESTRATIGRÁFICA }\end{array}$} & \multicolumn{3}{|c|}{ FECHADOS } \\
\hline & & Sigla & Años A.P. & Material \\
\hline Chorrillo Malo 2 & $75-85 \mathrm{~cm}$ nat & LP-502 & $1950 \pm 60$ & Carbón vegetal \\
\hline Co. Verlika 1 & $55-60 \mathrm{~cm}$ art & GX-25277 & $1685 \pm 70$ & Guanaco \\
\hline Co. Verlika 3 & & GX-27616 & $150 \pm 60$ & Carbón \\
\hline $\begin{array}{c}\text { Cueva } \\
\text { Lago Sofía } 1\end{array}$ & 2 & $\begin{array}{l}\text { OxA-9505 } \\
\text { OxA-9504 } \\
\text { OxA-8635 } \\
\text { OxA-9319 } \\
\text { AA-7283 }\end{array}$ & $\begin{array}{l}10140 \pm 120 \\
10310 \pm 160 \\
10710 \pm 70 \\
10780 \pm 60 \\
10910 \pm 260\end{array}$ & $\begin{array}{c}\text { Pseudalopex culpaeus } \\
\text { Onohipidium saldiasi } \\
\text { Guanaco } \\
\text { Onohipidium saldiasi } \\
\text { Carbón } \\
\end{array}$ \\
\hline $\begin{array}{c}\text { Cueva } \\
\text { Lago Sofía } 4 *\end{array}$ & & $\begin{array}{l}\text { PITT-0940 } \\
\text { AA-11498 }\end{array}$ & $\begin{array}{l}11590 \pm 100 \\
13.400 \pm 90\end{array}$ & $\begin{array}{l}\text { Mylodon sp. } \\
\text { Mylodon sp. }\end{array}$ \\
\hline $\begin{array}{c}\text { Cueva } \\
\text { Las Buitreras }\end{array}$ & Capa 2/3 & $\begin{array}{c}\text { CSIC-373 } \\
\text { Beta } 71985 \\
\text { Beta } 71984\end{array}$ & $\begin{array}{l}490 \pm 40 \\
670 \pm 60 \\
750 \pm 60\end{array}$ & $\begin{array}{c}\text { Carbón } \\
\text { Carbón vegetal } \\
\text { Carbón vegetal }\end{array}$ \\
\hline Cabo Vírgenes 8 & $65-70 \mathrm{~cm}$ & GX-25774 & $240 \pm 40$ & Guanaco \\
\hline \multirow{5}{*}{$\begin{array}{c}\text { Tres Arroyos } \\
1\end{array}$} & Nivel I & MC-1079 & $135 \pm 85$ & Carbón \\
\hline & Nivel III & Dic. 2731 & $700 \pm 70$ & Carbón \\
\hline & Nivel IV & Beta 30903 & $1340 \pm 50$ & Guanaco \\
\hline & $\begin{array}{l}\text { Nivel } \mathrm{Va} \\
\text { Nivel } \mathrm{Va} \\
\text { Nivel } \mathrm{Va} \\
\text { Nivel } \mathrm{Va} \\
\text { Nivel } \mathrm{Va} \\
\text { Nivel } \mathrm{Va} \\
\text { Nivel } \mathrm{Va}\end{array}$ & $\begin{array}{c}\text { OxA-9666 } \\
\text { Dic. } 2732 \\
\text { Beta } 113171 \\
\text { Beta } 101023 \\
\text { OxA-9246 } \\
\text { OxA-9247 } \\
\text { Beta } 20219 \\
\end{array}$ & $\begin{array}{l}10130 \pm 210 \\
10280 \pm 110 \\
10580 \pm 50 \\
10600 \pm 90 \\
10630 \pm 70 \\
10685 \pm 70 \\
11880 \pm 250\end{array}$ & $\begin{array}{c}\text { Carbón } \\
\text { Lama sp. } \\
\text { Fogón } \\
\text { Fogón } \\
\text { Vicugna sp. } \\
\text { Equidae } \\
\text { Óseo calcinado }\end{array}$ \\
\hline & Nivel V b & Dic. 2733 & $10420 \pm 100$ & Lama sp. \\
\hline San Julio 2 & Sondeo SJ1 & MC-1071 & Moderno & Carbón \\
\hline Cerro Sin Nombre & Capa C: 70-75 cm & Beta 116975 & $1150 \pm 60$ & Guanaco \\
\hline Cabeza de León 1 & Capa 2 & MC-1069 & $1100 \pm 95$ & Carbón vegetal \\
\hline
\end{tabular}

* El sitio Cueva Lago Sofía 4 es un conjunto paleontológico donde no se registró evidencia de actividad humana. Por el estudio de las propiedades de los hallazgos y su contexto, se ha interpretado al depósito como resultado de la actividad de una madriguera de un gran carnívoro (Pantera onca mesembrina; Borrero et al. 1997), aunque otros autores no consideran como probable que éste haya sido el agente acumulador (Tonni et al. 2003). 


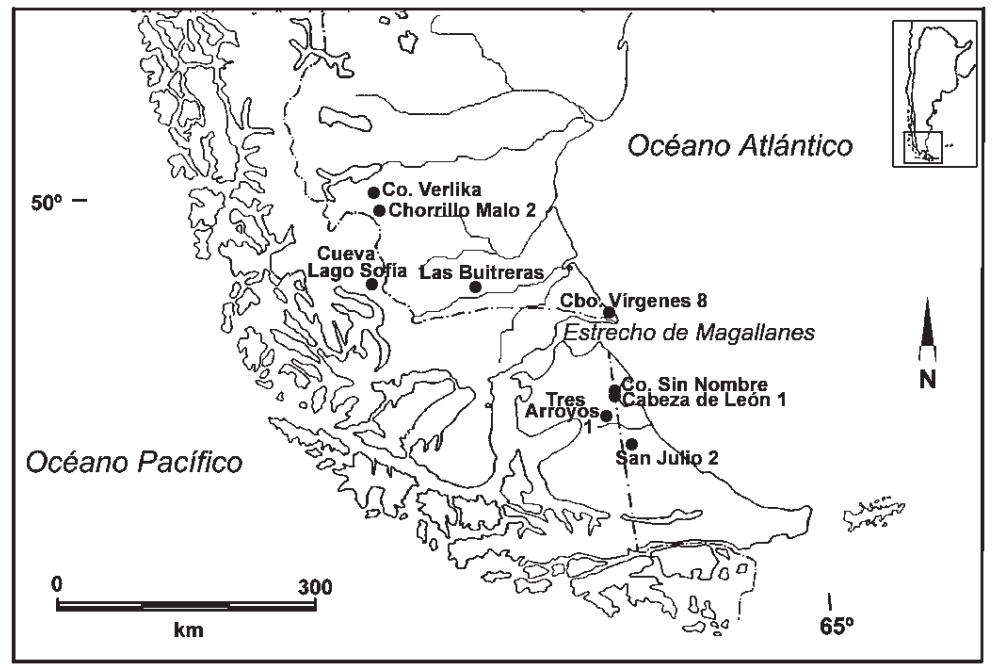

Fig. 1 Extremo austral de Patagonia continental e isla grande de Tierra del Fuego. Localización de los sitios analizados.

Para el conjunto de la isla de Tierra del Fuego, se utilizaron las muestras de los sitios: Tres Arroyos 1 (Borrero 2003; Massone 1987, Massone et al. 1993, 1998, 1999; Muñoz 1997, 2001, 2003; Mengoni Goñalons 1987); San Julio 2 (Horwitz et al. 1993/94); Co. Sin Nombre (Borrero 1985; Favier Duvois 2001; L'Heureux 1999c) y Cabeza de León 1 (Borrero 1979, 1985; Favier Duvois 2001). En la Tabla 1 se detallan las unidades estratigráficas consideradas para cada sitio, y los fechados radiocarbónicos disponibles para las mismas.

Las muestras estudiadas de la isla son casi exclusivamente tardías, las cuales fueron generadas de sitios y unidades estratigráficas correspondientes a los últimos 2.000 años (Tabla 1). Para este período temporal contamos con numerosos elementos óseos de guanaco, de los cuales se seleccionaron los húmeros distales y metacarpos distales por encontrarse bien representados en ambas regiones. En cambio, para el período correspondiente al Pleistoceno final, sólo contamos para las dos áreas con las $1^{\text {as }}$ falanges proximales y las $2^{\text {as }}$ falanges, en consecuencia, al analizar este período temporal sólo se utilizaron dichos elementos. En la Tabla 2 se detallan los totales de huesos medidos en cada área considerada y el bloque temporal al que adscriben. Se seleccionaron sólo los elementos con fusión completa, que no presentaban modificaciones pre o postdepositacionales que podían interferir en la obtención de la variable o hacer su registro poco confiable, y que registraran, como mínimo, el 80\% del total de variables medidas.

TABLA 2. Muestra analizada para cada área y unidad temporal.

\begin{tabular}{|c|c|c|c|}
\hline ÁREA & CRONOLOGÍA & HUESOS & ELEMENTOS MEDIDOS \\
\hline \multirow{6}{*}{$\begin{array}{c}\text { Patagonia } \\
\text { Continental } \\
\text { Austral }\end{array}$} & \multirow{2}{*}{$\begin{array}{l}\text { Pleistoceno final - } \\
\text { Holoceno temprano }\end{array}$} & Falange 1 proximal & 12 \\
\hline & & Falange 2 & 15 \\
\hline & \multirow{4}{*}{ Holoceno tardío } & Falange 1 proximal & 14 \\
\hline & & Falange 2 & 13 \\
\hline & & Húmero distal & 18 \\
\hline & & Metacarpo distal & 19 \\
\hline \multirow{6}{*}{$\begin{array}{l}\text { Patagonia } \\
\text { Insular }\end{array}$} & \multirow{2}{*}{$\begin{array}{l}\text { Pleistoceno final - } \\
\text { Holoceno temprano }\end{array}$} & Falange 1 proximal & 10 \\
\hline & & Falange 2 & 9 \\
\hline & \multirow{4}{*}{ Holoceno tardío } & Falange 1 proximal & 63 \\
\hline & & Falange 2 & 52 \\
\hline & & Húmero distal & 43 \\
\hline & & Metacarpo distal & 31 \\
\hline
\end{tabular}


$1^{\mathrm{a}}$ Falange. Epífisis Proximal

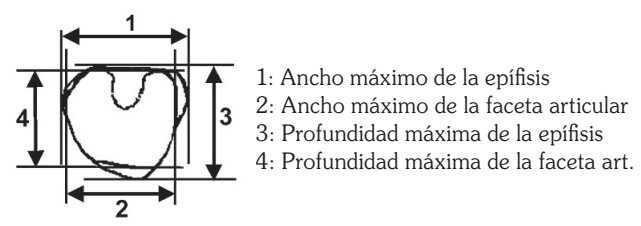

$2^{\text {a }}$ Falange

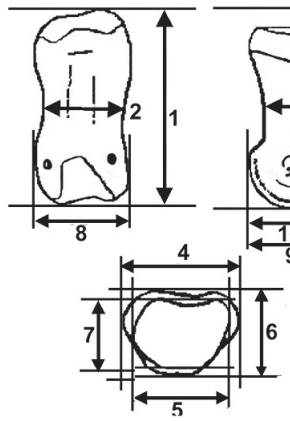

1: Longitud máxima

2: Ancho mínimo de la diáfisis

3: Profundidad mínima de la diáfisis

4: Ancho máximo de la ep. proximal

5: Ancho máx. de la faceta articular px.

Fig. 2 Variables osteométricas medidas en la epífisis proximal de la $1^{\text {a }}$ falange y en la $2^{\mathrm{a}}$ falange (vista proximal, anterior y lateral).

\section{ANÁLISIS OSTEOMÉTRICO}

El set de medidas registradas forma parte de una guía de mediciones especialmente adaptada a la anatomía del guanaco, la cual fue realizada en base a diferentes estándares previos (Kent 1982; Menegaz et al. 1988; Speth 1983; Von den Driesch 1976). Las medidas tomadas en cada elemento utilizado en este trabajo se representan en las Figs. 2 a 4 . La obtención de las variables continuas fue realizada con un calibre Vernier (precisión 0,01) y los datos registrados en $\mathrm{mm}$.

A partir del análisis métrico de elementos óseos, se obtuvo información relacionada con el tamaño y la forma de los elementos. Para inspeccionar
Húmero. Epífisis Distal

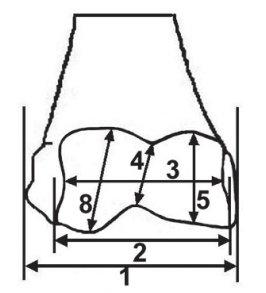

1: Ancho máximo de la epífisis

2: Ancho de la tróclea

3: Ancho mínimo de la tróclea

4: Altura central (mín.) de la tróclea

5: Altura máxima epicóndilo medial

6: Profundidad epicóndilo medial

7: Altura mínima epicóndilo lateral

8: Altura máxima epicóndilo lateral

9: Profundidad epicóndilo lateral
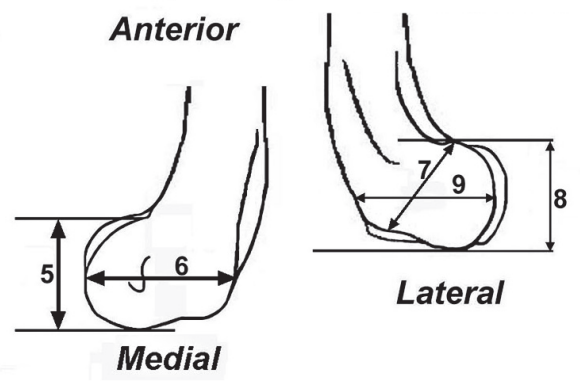

Lateral

Fig. 3 Variables osteométricas medidas en el húmero distal del guanaco. Vistas anterior, medial y lateral.

las variaciones asociadas con el tamaño, se utilizaron los valores crudos de las variables continuas obtenidas para cada hueso. En este caso, los test estadísticos aplicados fueron: análisis de varianza (ANOVA) y análisis de componentes principales (PCA). Los ANOVA presentados se realizaron sobre la Media Geométrica (MG) de cada elemento, utilizada en este trabajo como medida general del tamaño.

Para inspeccionar aspectos más relacionados con la forma, se aplicó el análisis discriminante (AD) con los datos estandarizados. Se utilizó la estandarización sugerida por Darroch y Mosimann (1985), obtenida a partir de la media geométrica. La totalidad de los análisis estadísticos se realizaron con los softwares: Stat Soft STATISTICA 6.0 y SYSTAT 10.2.

Metacarpo. Epífisis Distal

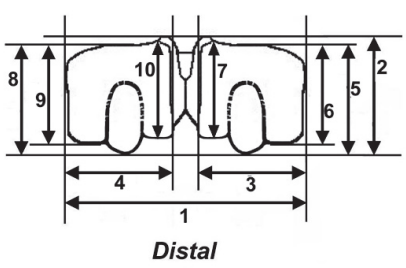

1: Ancho máximo de la epífisis

2: Profundidad máxima de la epífisis

3: Ancho del cóndilo medial

4: Ancho del cóndilo lateral

5: Profundidad reborde del cóndilo medial

6: Profundidad externa del cóndilo medial

7: Profundidad interna del cóndilo medial

8: Profundidad reborde del cóndilo lateral

9: Profundidad externa del cóndilo lateral

10: Profundidad interna del cóndilo lateral

Fig. 4 Variables osteométricas medidas en el metacarpo distal del guanaco. Vista distal. 
TABLA 3. Análisis de Varianza de la $2^{\mathrm{a}}$ Falange en Patagonia continental y Tierra del Fuego.

\begin{tabular}{|c|c|c|c|c|c|}
\hline Source & Suma de Cuadrados & $\mathrm{df}$ & Media de Cuadrados & F-ratio & $\mathrm{P}$ \\
\hline Área & 0.331 & 1 & 0.331 & 64.234 & 0.000 \\
\hline Error & 0.325 & 63 & 0.005 & & \\
\hline
\end{tabular}

Variable: Media Geométrica. Múltiple R: 0.711; Cuadrado múltiple R: 0.505.

En cuanto a la utilización de las falanges en el análisis morfológico, deben hacerse previamente algunas consideraciones. En material arqueológico es imposible discriminar, por lo menos de una forma confiable, las $1^{\text {as }}$ falanges proximales y las $2^{\text {as }}$ falanges (completas) procedentes de la pata delantera o trasera. En muestras actuales se ha demostrado la existencia de diferencias estadísticas significativas en el tamaño de las epífisis proximales de las $1^{\text {as }}$ falanges delanteras y posteriores (L'Heureux 2005), por lo que no se considera adecuado utilizar las $1^{\text {as }}$ falanges proximales sin discriminar para realizar estudios morfológicos basados en el tamaño de este elemento. En cambio, al utilizar los datos estandarizados, se observó la ausencia de diferencias entre las epífisis proximales de las $1^{\text {as }}$ falanges delanteras y posteriores. Por este motivo, en el presente trabajo sólo se presentarán los resultados de las variables estandarizadas de las $1^{\text {as }}$ falanges proximales. En cuanto a las $2^{\text {as }}$ falanges, en la muestra actual considerada, no se observaron diferencias entre las falanges delanteras y las posteriores al evaluarse tanto el tamaño como la forma (L'Heureux 2005), por lo que se utilizarán indistintamente en el presente trabajo.

Debido a la presencia de muestras con tamaños disimiles, p.e. los elementos del Holoceno tardío final de Tierra del Fuego frente a los otros conjuntos, se realizaron varios muestreos aleatorios

FALANGE $2^{\text {a }}$

ANOVA: Área + Tiempo

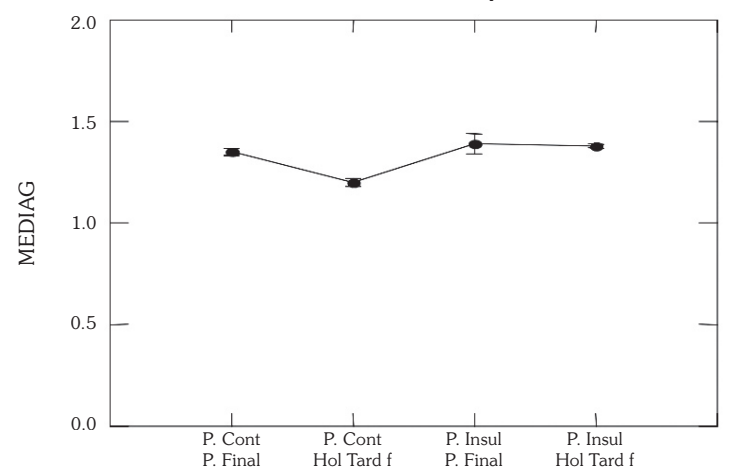

Fig. 5 Análisis de Varianza de la $2^{\mathrm{a}}$ falange en Patagonia continental y Tierra del Fuego por unidades temporales. dentro de las submuestras más grandes para hacer los conjuntos de huesos equivalentes. Los resultados obtenidos de los análisis con las muestras originales y los de las muestras aleatorizadas, fueron indistintos, por lo que se decidió presentar los resultados de los análisis con los tamaños muestrales originales presentados en Tabla 2.

\section{RESULTADOS}

\section{Falange $2^{a}$}

De acuerdo al análisis de varianza, se observa un aumento estadísticamente significativo en las dimensiones de las falanges $2^{\text {as }}$ de los guanacos de la isla, frente a las de la muestra del continente (P>0,01; Tabla 3). Si hacemos esta misma comparación teniendo en cuenta la variable temporal, se observa que en el Pleistoceno final no hay diferencias significativas en los tamaños de las $2^{\text {as }}$ falanges del continente y las de la isla, en cambio sí se observa en el Holoceno tardío final un gran incremento del tamaño en las muestras de la isla frente a las del continente ( $\mathrm{P}>0,01$; Fig. 5).

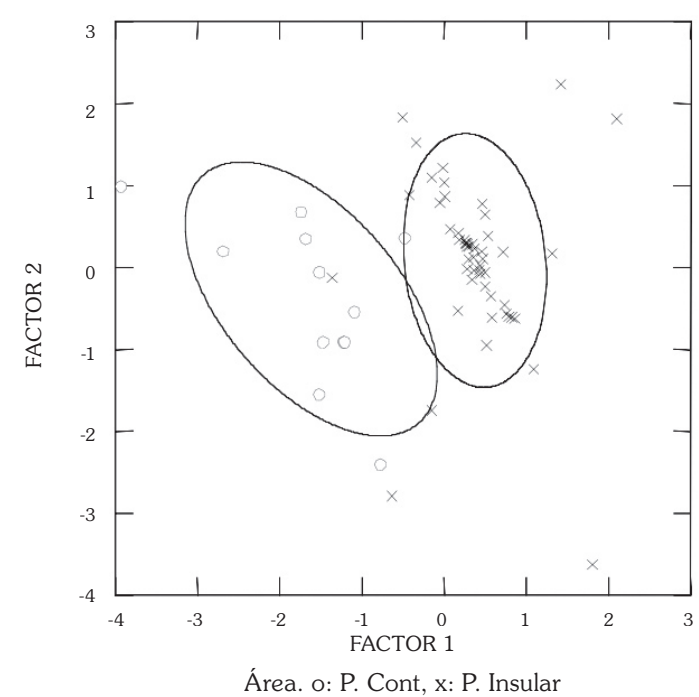

Fig. 6 Análisis de Componentes Principales de la $2^{\text {a }}$ falange por áreas. 


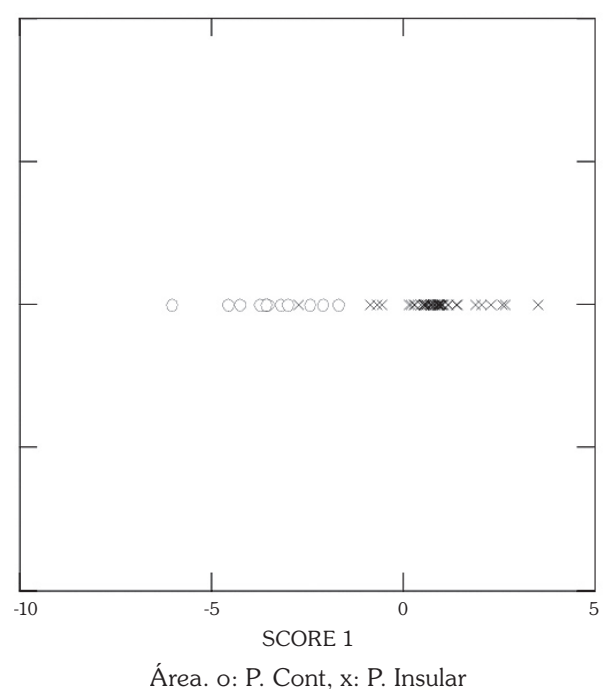

Fig. 7 Análisis Discriminante con variables estandarizadas de la $2^{a}$ falange por áreas.

Si analizamos la información en conjunto a partir de un método de reducción de variables (PCA), observamos que según el Factor 1, las muestras de la isla se separan de las del continente (Fig. 6). Esto se relaciona con la presencia de tamaños mayores en Tierra del Fuego. Si se utilizan los datos estandarizados para explorar comportamientos relacionados con la forma del hueso a partir de un análisis discriminante, se obtiene una distribución diferencial entre las formas de las falanges $2^{\text {as }}$ de la

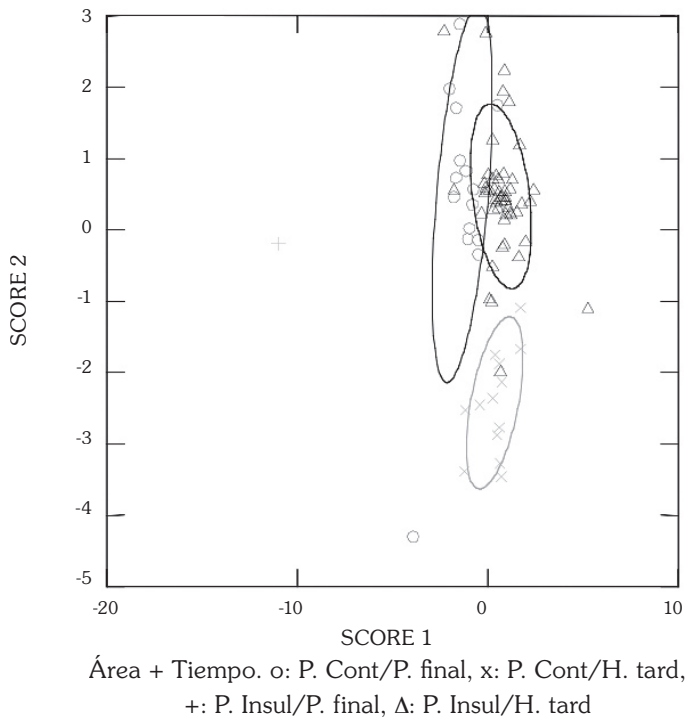

Fig. 9 Análisis discriminante con variables estandarizadas de la $2^{a}$ falange por áreas y unidades temporales.

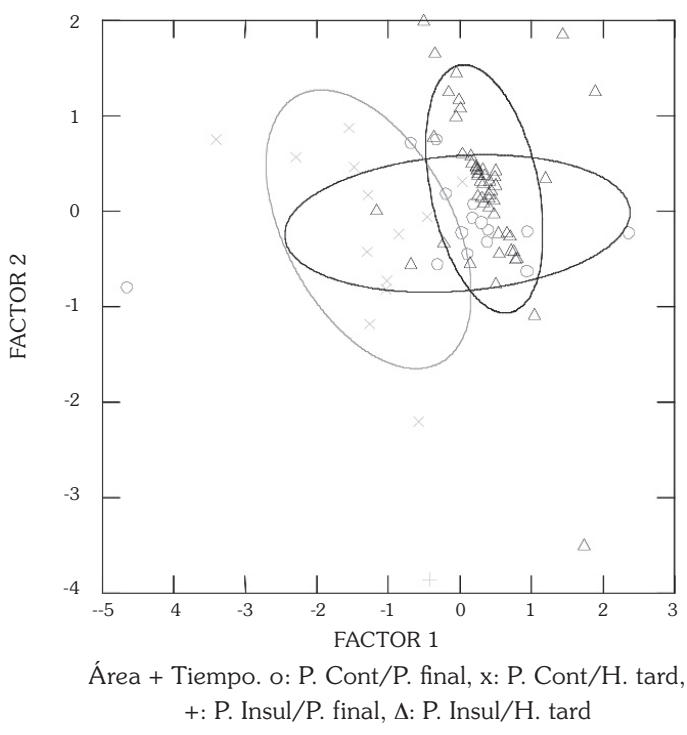

Fig. 8 Análisis de componentes principales de la $2^{\mathrm{a}}$ falange por áreas y unidades temporales.

isla y las del continente $(\mathrm{P}=0,000$; Lambda de Wilks: 0,26; Fig. 7), dado por un solo Score.

Al incorporar la variable temporal al análisis de PCA, tenemos en el Factor 1 una clara diferenciación en el tamaño en el Holoceno tardío final entre los elementos del continente y los de la isla, quedando el conjunto de las $2^{\text {as }}$ falanges de fines del Pleistoceno en una situación intermedia (Fig. 8). Un comportamiento similar se observa en el análisis discriminante para detectar comportamientos asociados con la forma: las muestras del Holoceno tardío se diferencian en el eje de las Y, y la muestra del Pleistoceno final no resulta distinguible de los otros grupos (P-tail = 0,000; Lambda de Wilks: 0,08; Fig. 9).

A partir de la información expuesta, se observa que para las $2^{\text {as }}$ falanges hay cambios morfológicos importantes relacionados con una variación geográfica y temporal. Si comparamos los conjuntos del Holoceno tardío, el tamaño de los elementos aumenta notablemente entre las muestras de la isla frente a las del continente, pero si comparamos las muestras de fines del Pleistoceno, no se registran diferencias en los tamaños ni los aspectos relacionados con la forma de las $2^{\text {as }}$ falanges del continente y de la isla. En este sentido, vemos que las muestras son morfológicamente similares a un lado y otro del Estrecho hasta el Holoceno temprano, y se genera una diferenciación que resulta estadísticamente significativa hacia fines del Holoceno tardío. 


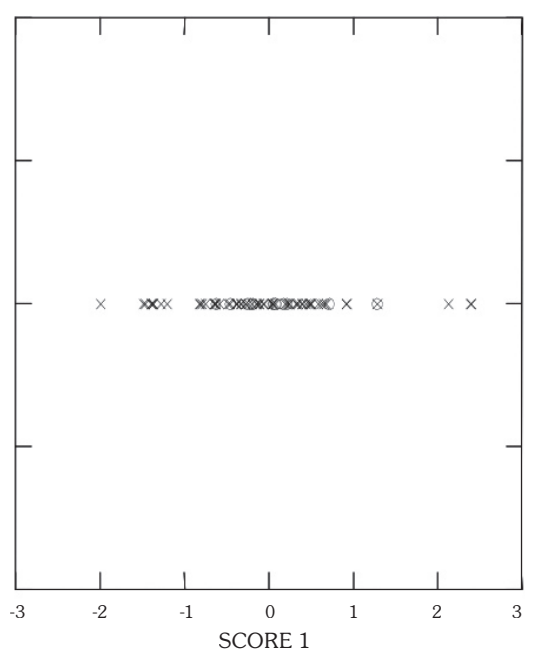

Fig. 10 Análisis discriminante con variables estandarizadas de la $1^{\text {a }}$ falange proximal por áreas y unidades temporales.

\section{Falange $1^{a}$. Epífisis proximal}

El análisis discriminante de los datos estandarizados de las primeras falanges proximales, no registra diferencias en los aspectos asociados con la forma del hueso entre las muestras de Patagonia austral y las de Tierra del Fuego (P-tail: 0,55; Lambda de Wilks: 0,9590).

Al considerar los distintos períodos temporales, se observa que las muestras de fines del Pleistoceno de ambas regiones tienden a diferenciarse de aquellas del Holoceno tardío final a partir del Score 1, pero esta tendencia no se traduce en una diferencia estadísticamente significativa (P-tail: 0,000; Lambda de Wilks: 0,665; Fig. 10). A partir de lo expuesto, vemos que en este hueso no se observan diferencias geográficas estadísticas asociadas con la forma, y sólo se registra una tendencia temporal a diferenciarse, pero sin llegar a hacerlo en forma significativa. Se confirma la similitud relacionada en los aspectos de la forma entre las $1^{\text {as }}$ falanges proximales del Pleistoceno final de ambas regiones.

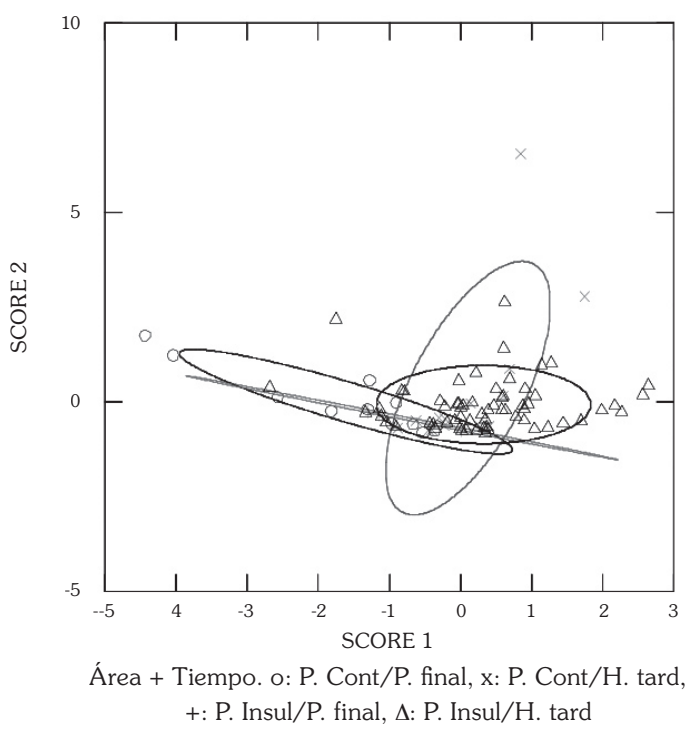

Fig. 11 Análisis de componentes principales del húmero distal por áreas.

\section{Húmero Distal}

Los húmeros distales sólo pudieron ser analizados en el bloque temporal correspondiente al Holoceno tardío final. De acuerdo al análisis de comparación de medias, vemos un incremento en el tamaño estadísticamente significativo en el conjunto de Tierra del Fuego frente al de Patagonia austral ( $\mathrm{P}>0,001 ;$ Tabla 4). Según el análisis de PCA, los conjuntos se diferencian en el tamaño a partir del Factor 1, siendo la muestra de la isla más heterogénea (Fig. 11). Teniendo en cuenta los datos estandarizados, el análisis discriminante exhibe aspectos relacionados con la forma diferentes para las muestras de la isla y para las muestras de Patagonia austral continental (P-tail: 0,001; Lambda de Wilks: 0,54; Fig. 12), resumidas en un solo Score.

En suma, en la epífisis distal del húmero se registran diferencias geográficas importantes. Es notable el incremento de tamaño y de robustez sufrido en el conjunto de la isla hacia fines del Holoceno tardío, frente al conjunto del extremo austral de Patagonia continental del mismo período temporal.

TABLA 4. Análisis de Varianza del Húmero distal de Patagonia Continental y Tierra del Fuego.

\begin{tabular}{|c|c|c|c|c|c|}
\hline Source & Suma de Cuadrados & $\mathrm{df}$ & Media de Cuadrados & F-ratio & $\mathrm{P}$ \\
\hline Áreas & 0.877 & 1 & 0.877 & 12.755 & 0.001 \\
\hline Error & 3.301 & 48 & 0.069 & & \\
\hline
\end{tabular}

Variable: Media Geométrica. Múltiple R: 0.458. Cuadrado múltiple R: 0.210. 
TABLA 5. Análisis de Varianza del Metacarpo distal de Patagonia Continental y Tierra del Fuego

\begin{tabular}{|c|c|c|c|c|c|}
\hline Source & Suma de Cuadrados & $\mathrm{df}$ & Media de Cuadrados & F-ratio & $\mathrm{P}$ \\
\hline Áreas & 0.202 & 1 & 0.202 & 19.751 & 0.000 \\
\hline Error & 0.337 & 33 & 0.010 & & \\
\hline
\end{tabular}

Variable: Media Geométrica. Múltiple R: 0.612. Cuadrado múltiple R: 0.374

\section{Metacarpo distal}

Este elemento también es analizado en un solo bloque temporal, el cual se restringe al Holoceno tardío final. De acuerdo al análisis de comparación de medias, vemos un aumento en el tamaño estadísticamente significativo entre las regiones $(\mathrm{P}>0,001)$, siendo considerablemente mayor en los elementos de la isla de Tierra del Fuego (Tabla 5). Analizando el tamaño a partir de componentes principales, se observa la separación de las muestras de uno y otro lado del Estrecho siguiendo el Factor 1 (Fig. 13). Para inspeccionar aspectos relacionados con la forma, vemos que el análisis discriminante separa los metacarpos distales de la isla de aquéllos procedentes del extremo más austral del continente (P-tail: 0,000; Lambda de Wilks: 0,29; Fig. 14), lo cual se relaciona con la presencia de formas más robustas en la isla.

En general, se observa una variación morfológica entre los elementos de Patagonia austral y aquéllos procedentes de la isla grande de Tierra del

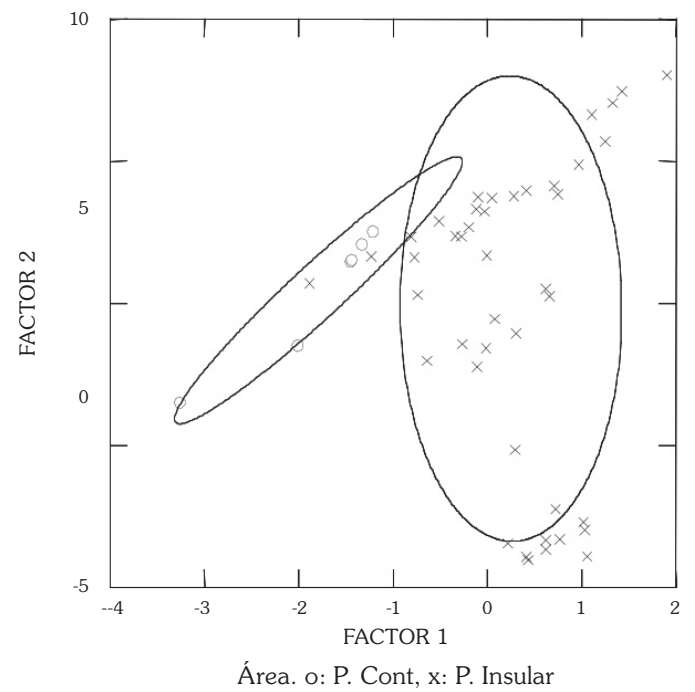

Fig. 12 Análisis discriminante con variables estandarizadas del húmero distal por áreas.
Fuego durante el Holoceno tardío final. Estas diferencias se relacionan con un incremento significativo del tamaño y una mayor robustez de los elementos de la isla en el mismo período temporal.

\section{DISCUSIÓN}

Como fuera mencionado anteriormente, el tamaño del cuerpo de los guanacos tiene relevancia teniendo en cuenta la estrecha relación que contemplan los aspectos fenotípicos del animal con su adaptación al medio, ya sea por concederles ventajas selectivas frente a la apropiación de recursos o en el mecanismo de huida de los depredadores, etc. A pesar de que el fenotipo de un animal es el resultado de un compromiso entre muchas y conflictivas presiones selectivas (Mayr 1956), y la evaluación del tamaño corporal no es un objetivo simple que se desprende en forma directa del tamaño de los elementos óseos, intentamos aproximarnos en forma indirecta a dicho principio a partir de la información obtenida de los tamaños de restos óseos arqueológicos de guana-

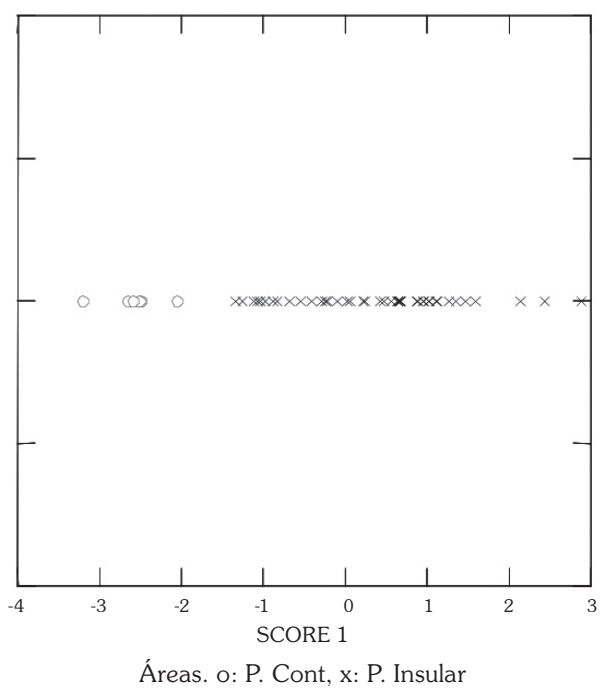

Fig. 13 Análisis de componentes principales del metacarpo distal por áreas. 


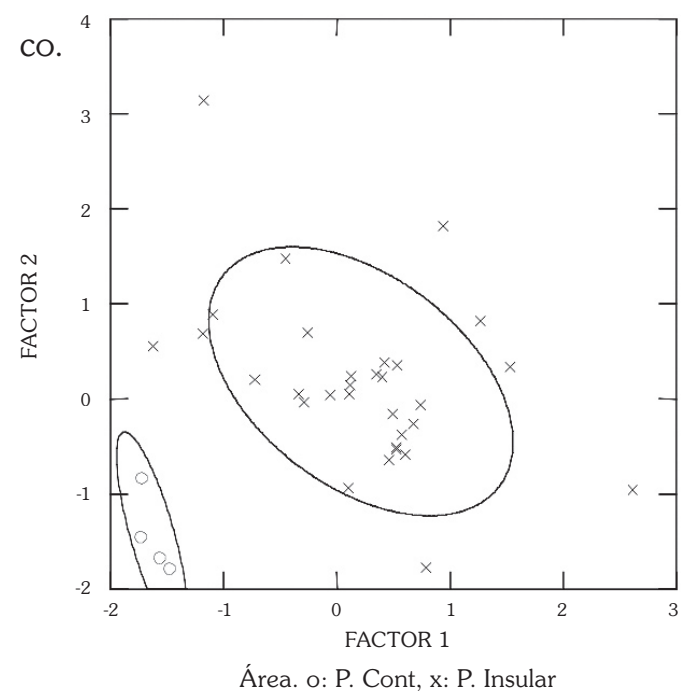

Fig. 14 Análisis discriminante con variables estandarizadas del metacarpo distal por áreas.

Por otro lado, vemos que el caso estudiado presenta características ecológicas y geográficas particulares que exigen un tratamiento profundo. Estamos comparando dos regiones con historias biogeográficas diferentes. En un acápite previo se comentó que el actual extremo sur continental y la isla grande de Tierra del Fuego hacia fines del Pleistoceno y principios del Holoceno formaban parte de un área original continua. De acuerdo a esto nos encontramos ante un panorama que presenta una distribución "ininterrumpida" (dentro de los territorios libres de hielo y de las características discontinuas de los recursos y los grupos humanos), de las poblaciones de animales en toda el área de estudio hasta el Holoceno temprano.

Luego, a partir de importantes modificaciones climáticas y geomorfológicas acontecidas a escala suprarregional, se sucede un evento de vicarianza acarreando importantes consecuencias en el ambiente y en los taxa que habitaban el área. Evidencia geológica ubica la aparición de la barrera natural (el estrecho de Magallanes), hacia ca. 8.000 años AP (McCulloch et al. 1997; Rabassa et al. 1992a,b). A partir de la instauración de la barrera marina y la disrrupción en la extensión original (y continua) del taxón ancestral, se sucedieron una serie de procesos que llevaron a la divergencia de las trayectorias evolutivas del taxón ancestral y el taxón vicariante. Como fuera planteado por Borrero (1989/1990) para el caso de las poblaciones humanas, al implantarse el
Estrecho debió ocurrir un completo reordenamiento del espacio explotado produciéndose procesos de contracción y expansión de los rangos de acción de las poblaciones instaladas cerca del Estrecho. Como parte de dicho reordenamiento debió comenzar un proceso de evolución independiente (Borrero 1989/1990).

En este mismo sentido, se plantea un caso de vicarianza y posterior evolución divergente para las poblaciones de guanaco, generando diferencias en las trayectorias biológicas de dichas poblaciones a un lado y otro del estrecho de Magallanes. Las diferencias seguramente fueron incrementándose al transcurrir las generaciones, a partir del proceso de aislamiento - efecto fundador y deriva génica. Como consecuencia de la acción de este mecanismo evolutivo, la variabilidad y el pool génico de la población de guanacos debió tender a la reducción por aislamiento y, en consecuencia, ciertos alelos pudieron fijarse en el genotipo y expresarse en el fenotipo por azar y no por adaptabilidad (L'Heureux 2004). La evidencia morfológica presentada apunta hacia una selección de tamaños grandes y formas robustas en las poblaciones de la isla desde fines del Pleistoceno hasta el presente, y una reducción de los tamaños en el continente durante el Holoceno.

Por otro lado, diferentes cuerpos de datos independientes como la evidencia antropocultural (Borrero 1989/1990), bioantropológica (Cocilovo y Guichón 1985-1986) y genética de poblaciones de guanaco (Sarno et al. 2000 para datos moleculares), sugieren que las trayectorias evolutivas biológicas y culturales al norte y sur del estrecho de Magallanes, tuvieron cierta profundidad temporal y cierta independencia (Borrero 1989/1990).

Concluyendo, se considera que la información morfológica presentada aporta nuevos datos para discutir el proceso de evolución divergente entre Patagonia austral continental y la isla grande de Tierra del Fuego. El patrón obtenido apunta hacia una diferenciación en el tamaño y en la forma de los huesos de guanaco posterior al Holoceno temprano y que resulta estadísticamente significativa hacia el Holoceno tardío final. La diferenciación morfológica se produjo una vez instaurada la barrera natural y generó fenotipos -evaluados a partir de los tamaños y la forma de los huesos de guanaco- diferentes a un lado y otro del Estrecho. Esto concuerda con el planteo de evolución divergente propuesto para 
Tierra del Fuego por Borrero (1989/1990) para poblaciones humanas. En este sentido, considero que las diferencias en los patrones morfológicos observados se relacionan con las diferencias en las trayectorias biológicas de las poblaciones de guanaco procedentes de ambos lados del estrecho de Magallanes.

\section{AGRADECIMIENTOS}

Agradecemos a Mateo Martinic, Mauricio Massone y Alfredo Prieto por permitir gentilmente acceder y analizar las muestras de TA1 y CLS depositadas en el laboratorio de Arqueología del Centro de Estudios del Hombre Austral del Instituto de la Patagonia, Universidad de Magallanes, Punta Arenas. A Pedro Cárdenas por la ayuda brindada durante mi estadía en el mencionado laboratorio. A la Dra. M. Amalia Sanguinetti de Bórmida por permitirme estudiar las muestras de cueva Las Buitreras. A Luis A. Borrero por los comentarios realizados que ayudaron al mejoramiento del manuscrito.

Este trabajo se realizó dentro del marco de los proyectos PIP-CONICET 4536-96 "Magallania II" y PICT-ANPCyT 04-00807 "Arqueología distribucional en escala supraregional".

\section{BIBLIOGRAFÍA}

ASHTON, K.G., TRACY, M.C. y A. DE QUEIROZ. 2000. Is Bergmann's rule valid for mammals? American Naturalist 156, 390-415.

ATKINSON, D. 1994. Temperature and organism size - a biological law for ectotherms? Advance in Ecolological Research, 25, 1-58.

BERGMANN, C. 1847. Ü ber die Verhaltnisse der Warmeokonomie der Thiere zu ihrer Grosse. Go"ttingen Studien, Part 1:595-708.

BORRERO, L. A. 1979. Excavaciones en el alero "Cabeza de León". Isla Grande de Tierra del Fuego. Relaciones de la Sociedad Argentina de Antropología XIII: 255-271.

- 1985. La Economía Prehistórica de los Habitantes del Norte de la Isla Grande de Tierra del Fuego. Tesis Doctoral inédita, Universidad de Buenos Aires.

- 1989-1990. Evolución cultural divergente en la Patagonia austral. Anales del Instituto de la Patagonia, Serie Ciencias Sociales, 19: 133-139.

- 2001. El Poblamiento de la Patagonia. Toldos, milodones y volcanes. Emecé.

- 2003. Taphonomy of the Tres Arroyos 1 Rockshelter, Tierra del Fuego, Chile. Quaternary International 109110: 87-93.
BORRERO, L. A. y N. V. FRANCO. 1999. Arqueología de Cabo Vírgenes, Provincia de Santa Cruz. En: Actas del XIII Congreso Nacional de Arqueología Argentina, Córdoba, en prensa.

BORRERO, L. A., F. M. MARTIN y A. PRIETO. 1997. La Cueva Lago Sofía 4. Una madriguera Pleistocénica. Anales del Instituto de la Patagonia, Serie Ciencias Sociales 25:103-122, Punta Arenas.

BROWN, J. H. y K. LEE. 1969. Bergmann's rule and climatic adaptation woodrats (Neotoma). Evolution 23:329338.

CAVIGLIA, S. E. y M. J. FIGUERERO TORRES. 1976. Material faunístico de la cueva "Las Buitreras" (Dpto. Güer Aike, Provincia de Santa Cruz). En: Relaciones de la Sociedad Argentina de Antropología X:315-319, Buenos Aires.

COCILOVO, J. A. y R. A. GUICHÓN. 1985-1986. Propuesta para el estudio de las poblaciones aborígenes del extremo austral de Patagonia. Anales del Instituto de la Patagonia, Serie Ciencias Sociales 16:111-123, Punta Arenas.

CRICI, J. V. y J. J. MORRONE. 1990. En busca del paraíso perdido: la biogeografía histórica. Ciencia Hoy 1(5): 25-34.

DAVIS, S. M. J. 1981. The effects of temperature change and domestication on the body size of Late Pleistocene to Holocene mammals of Israel. Paleobiology 7(1):101114.

DARROCH, J. N. y J. E. MOSIMANN. 1985. Canonical and principal component of shape. Biométrika 72:241252.

FAVIER DUVOIS. 2001. Análisis Geoarqueológico de los procesos de formación del registro, cronología y paleoambientes, en sitios arqueológicos de Fuego-Patagonia. Tesis Doctoral inédita. Facultad de Ciencias Exactas y Naturales. UBA.

FORSMAN, A. 1991. Variation in sexual size dimorphism and maximun body size among adder populations: effects of prey size. Journal of Animal Ecology 60:253-267.

FRANCO, N. V. 2002. Estrategias de utilización de recursos líticos en la cuenca superior del río Santa Cruz. Tesis doctoral. Universidad Nacional de Buenos Aires.

FRANCO, N. V. y L. A. BORRERO. 2003. Chorrillo Malo 2: initial peopling of the upper Santa Cruz basin, Argentina. En Where the South Winds Blow. Ancient Evidence of Paleo South Americans. Editado por R. Bonnischsen, L. Miotti, M. Salemme y N. Flegenheimer, pp.141-147. Center for the Studies of the First Americans (CSFA) y Texas A\&M University Press.

FRANCO, N.V., L.A. BORRERO, J.B. BELARDI, F. CARBALLO MARINA, F.M. MARTIN, P. CAMPAN, C. FAVIER DUBOIS, N. STADLER, M.I. HERNÁNDEZ LLOSAS, H. CEPEDA, A.S. MUÑOZ, F. BORELLA, F. MUÑOZ e I. CRUZ. 1999. Arqueología del Cordón Baguales y sistema lacustre al sur del Lago Argentino (provincia de Santa Cruz, Argentina). Praehistoria 3: 65-86, PREP.

HAYWARD, J. S. 1956. Microclimate temperature and its adaptative significance in six geographic races of Peromyscus. Canadian Journal of Zoology 43:341-350. 
HORWITZ, V., L. A. BORRERO, y M. CASIRAGHI. 1993-94. San Julio 2 (Tierra del Fuego). Estudios del Registro Arqueológico. Relaciones de la Sociedad Argentina de Antropología XIX:391-415.

JAMES, F.C. 1970. Geographical size variation in birds and its relationship to climate. Ecology 51, 365-390.

KENT, J. K. 1982. The Domestication and Exploitation of the South American Camelids: Methods of Analysis and their Application to Circum-lacustrine Archaeological Sites in Bolivia and Peru. Tesis Doctoral inédita, St. Louis, Washington University.

L'HEUREUX, G.L. 1999a. Análisis arqueofaunístico del sitio Cerro Verlika 3. Informe interno. Programa de Estudios Prehistóricos (PREP). MS.

- 1999b. Análisis arqueofaunístico del sitio Cabo Vírgenes 8. Informe interno. Programa de Estudios Prehistóricos (PREP). MS.

- 1999c. Análisis arqueofaunístico del sitio Cerro Sin Nombre. Informe interno. Programa de Estudios Prehistóricos (PREP). MS.

- 2004. Estudio arqueológico del proceso coevolutivo entre las poblaciones humanas y las poblaciones de guanacos en Magallania (Patagonia meridional y norte de Tierra del Fuego). En prensa en las Actas del XIV Congreso Nacional de Arqueología Argentina, Universidad Nacional de Rosario.

- 2005. El estudio arqueológico del proceso coevolutivo entre las poblaciones humanas y las poblaciones de guanacos en Patagonia Meridional y Tierra del Fuego. Tesis Doctoral en preparación. Facultad de Ciencias Naturales y Museo, Universidad Nacional de La Plata.

MARKGRAF, V. 1988. Fell's Cave: 11,000 years of changes in paleoenvironments, fauna and human ocupation. Travels and Archaeology in South Chile, editado por J. Hyslop, pp. 196-201. University of Iowa Press.

- 1989. Paleoclimates in Central and South America since 18,000 BP based on pollen and lake-level recors. Quaternary Sciences Reviews 8:1-24.

MASSONE, M. 1987. Los cazadores paleoindios de Tres Arroyos (Tierra del Fuego). Anales del Instituto de la Patagonia, Serie Ciencias Sociales 17:47-60, Punta Arenas.

MASSONE, M., D. JACKSON y A. PRIETO. 1993. Perspectivas arqueológicas de los Selk'nam. Colección de Antropología. Centro de Investigación Diego Barros Arana, Santiago de Chile.

MASSONE, M., A. PRIETO, D. JACKSON y M. A. ARROYO. 1998. Los cazadores tempranos y el fuego: nuevos antecedentes del sitio Tres Arroyos 1. Trabajo presentado a las Cuartas Jornadas de Arqueología de la Patagonia, Río Gallegos, Argentina.

MASSONE, M., A. PRIETO, D. JACKSON, X. PRIETO, G. ROJAS, R. SEGUEL y LUIS BORRERO. 1999. Hombre temprano y Paleoambiente en Tierra del Fuego. Informe de avance $3^{\circ}$ Año, FONDECYT N ${ }^{\circ} 1960027$. Universidad de Magallanes; Universidad de Chile.

MAYR, E. 1956. Geographical character gradients and climatic adaptation. Evolution 10, 105-108.

MCCULLOCH, R., C. CLAPPERTON, J. RABASSA y A. CURRANT. 1997. The glacial and Postglacial environmental history of Fuego-Patagonia. En: Natural History, Prehistory and Etnography at the Uttermost End of the Earth editado por C. McEwan, L. A. Borrero y A. Prieto, pp.12-31. British Museum Press, London

MCNAB, B. 1971. On the ecological significance of Bergmann's rule. Ecology 52, 845-854.

MENEGAZ, A., M.C. SALEMME y E. ORTIZ JAUREGUIZAR. 1988. Una propuesta de sistematización de los caracteres morfométricos de los metapodios y las falanges de camelidae. En: N. Ratto y A. Haber (eds) De procesos, Contextos y otros Huesos, Buenos Aires: FFyL-UBA pp. 53-64.

MENGONI GOÑALONS, G. 1987. Modificaciones culturales $y$ animales en los huesos de los niveles inferiores del sitio Tres Arroyos 1 (Tierra del Fuego, Chile). Anales del Instituto de la Patagonia, Serie Ciencias Sociales 17:61-66, Punta Arenas.

MOUSSEAU, T.A. 1997. Ectotherms follow the converse to Bergmanns's rule. Evolution, 51, 630-632.

MUÑOZ, A.S. 1997. Explotación y procesamiento de ungulados en Patagonia meridional y Tierra del Fuego, Anales del Instituto de la Patagonia, Serie Ciencias Sociales 25:201-222, Punta Arenas.

- 2001. El guanaco en la dieta de los cazadores-recolectores del norte de Tierra del Fuego. Análisis de dos casos procedentes del área Bahía Inútil-San Sebastián. En: El uso de los Camélidos a través del Tiempo, (ed. por G.L. Mengoni Goñalons, D.E. Olivera y H.D. Yacobaccio), pp. 155-178. GZC-ICAZ, Ediciones del Tridente, Buenos Aires.

- 2003. La Explotación de Mamíferos por CazadoresRecolectores Terrestres de Tierra del Fuego. Tesis de Doctorado. Facultad de Filosofía y Letras, Universidad Nacional de Buenos Aires.

PLATNICK, N. I. y G. NELSON. 1978. A method of analysis for historical biogeography. Systematic Zoology 27:1-16.

PRIETO, A. 1991. Cazadores tempranos y tardíos en la cueva Lago Sofía 1. Anales del Instituto de la Patagonia, Serie Ciencias Sociales 20:75-100, Punta Arenas.

RABASSA, J. y C. M. CLAPPERTON. 1990. Quaternary glaciations of the Southern Andes. Quaternary Science Reviews 9:153-174.

RABASSA, J., C.J. HEUSSER y N. RUTTER. 1992a. El Tardiglacial y Holoceno de Tierra del Fuego. El Holoceno en la Argentina, editado por Iriondo, pp. 103-120. CADINQUA, Paraná.

RABASSA, J., G. BUJALESKI, A. MEGLIOLI, A. CORONATO, S. GORDILLO, C. ROIG y M. SALEMME. 1992b. The Quaternary of Tierra del Fuego: the status of our knowledge. Sveriges Geologiska Undersökning 81:249-256.

RAY, C. 1960. The application of Bergmann's rule and Allen's rule to the poikilotherms. Journal of Morphology, 106, 85-109.

ROFF, D. 1980. Optimizing development time in a seasonal environment: the ups and downs of clinal variation. Oecologia 45, 202-208.

- 1992. The evolution of life histories: Theory and Analisis. Chapnam Hall Inc., New York.

RONQUIST, F. 1997. Dispersal-vicariance analysis: a new approach to the quantification of historical biogeography. Systematic Biology 46: 195-203. 
RONQUIST, F. y S. NYLIN. 1990. Process and pattern in the evolution of species associations. Systematic Zoology 39: 329-344.

ROSEN, D. E. 1978. Vicariant patterns and historical explanation in biogeography. Systematic Zoology 27:159-188.

ROSENZWEIG, M. L. 1966. Community structure in Sympatric carnivora. Journal of Mammology 47:602-612

SANGUINETTI DE BÓRMIDA, A. 1976. Excavaciones prehistóricas en la cueva de "Las Buitreras" (Provincia de Santa Cruz). En Relaciones de la Sociedad Argentina de Antropología X:271-292, Buenos Aires.

- 1980. El sitio las Buitreras como aporte al manejo de fuentes prehistóricas del temprano poblamiento sudamericano. RUNA XIII (1-2):11-20, Buenos Aires.

- 1999. Informe sobre las investigaciones llevadas a cabo en el área de Investigaciones de la cuenca del Río Gallegos (Provincia de Santa Cruz, Argentina). En: Praehistoria 3:119-133, Buenos Aires.

SARNO, R. J., W. L. FRANKLIN, S. O'BRIEN y W. E. JOHNSON. 2000. Uso de marcadores genéticos para la conservación de los camélidos sudamericanos silvestres. En: Manejo Sustentable de la vicuña y el guanaco.
Editado por: B. Gonzáles, P. Bas, Ch. Tala y A. Iriarte, pp. 27-44. Servicio Agrícola y Ganadero. Pontificia Universidad Católica de Chile, Fundación para la Innovación Agraria, Chile.

SCHOLANDER, P. F. 1955. Evolution of climatic adaptation in homeoterms. Evolution 9:15-26.

SPETH, J.D. 1983. Bison Kills and Bone Counts. Decision Making by Ancient Hunters. Prehistoric Archaeology and Ecology. Serie editada por K.W. Butzer y L.G. Freeman. University of Chicago Press.

STINE, S. y M. STINE. 1990. A record from Lake Cardie of Climate Change in Southern America. Nature 345:705-708.

TONNI, E. P., A. A. CARLINI, G. J. SILICATO YAÑÉ y A. J. FIGINI. 2003. Cronología radiocarbónica y condiciones climáticas en la Cueva del Milodón (sur de Chile) durante el Pleistoceno Tardío. Ameghiniana, 40(4):609-615.

VON DEN DRIESCH, A. 1976. A guide to the measurement of animal bones from archaeological sites, Peabody Museum Bulletins (1). Harvard University.

WILEY, E. O. 1988. Vicariance biogeography. Annual Review of Ecology and Systematics 19:513-542. 